\title{
Ultraestructura de hongos encontrados en la atmósfera de Santafé de Bogotá
}

\author{
José B. Yung ${ }^{1}$, María C. Cepero², Francisco J. Leal ${ }^{3}$
}

\begin{abstract}
Resumen
Se describen las características morfológicas de esporulación al microscopio electrónico de rastreo (MER) de algunos de los principales hongos encontrados en la atmósfera de Santafé de Bogotá. Los hongos se fijaron con vapores de acroleína pura y cristales de $\mathrm{OsO}_{4}$ y se secaron en alto vacío, que evita el daño de las estructuras aéreas y arreglos característicos, como lo hemos informado previamente. El análisis al MER reveló la variedad morfológica de los hongos estudiados y permitió observar con detalle las características ultraestructurales de la superficie de los hongos, lo cual es de importancia en la realización del estudio morfológico, estructural y taxonómico; reveló estructuras y características morfológicas y detalles de la conidiogénesis que ayudan a un mejor entendimiento del proceso; algunos de estos detalles estructurales son difíciles de observar al microscopio de luz o al microscopio de contraste de fase.
\end{abstract}

\section{Summary}

Morphological sporulation characteristics of some of the chief potential allergenic molds found in the atmosphere of Santafé de Bogotá, were observed by scanning electron microscopy. The molds were fixed in pure acrolein vapors and $\mathrm{OsO}_{4}$ crystals and were dried in high vacuum to avoid damage to aerial structures and characteristic arrangements, as we have previously reported. SEM analysis revealed morphological variations of the studied molds and allowed detailed observation of their surface ultrastructural characteristics, being of importance in the development of a structural, morphological and taxonomical study, by revealing structures, morphological characteristics and conidiogenetic details to provide better understanding of the process. Some structural details are difficult to observe by light microscope or phase-contrast microscope.

Los hongos se encuentran distribuidos en la naturaleza en el suelo, asociado con material orgánico o transitoriamente en el aire. Muchos de los hongos ambientales no patógenos para el hombre se denominan contaminantes. Algunos de éstos se asocian a reacciones alérgicas y cada vez se tiene más evidencia de la importancia de estos hongos en la etiología alérgica como res- puesta al contacto e inhalación de esporas encontrados en el aire (3-4). El estudio microscópico para determinar las características de la conidiogénesis debe hacerse a partir de microcultivos ya que, en preparaciones a partir de cultivos, las estructuras se distorsionan fácilmente, observándose únicamente gran cantidad de conidios libres.

1 Laboratorio de Investigación Clínica, Departamento de Ciencias Biológicas, Universidad de Los Andes; Laboratorio de Inmunología, Hospital de San José, Santafé de Bogotá, D.C.

2 Departamento de Ciencias Biológicas, Centro de Investigaciones Microbiológicas, Universidad de Los Andes, Santafé de Bogotá, D.C.

3 Unidad de Alergia e Inmunología, Hospital Infantil Universitario Lorencita Villegas de Santos, Santafé de Bogotá, D.C. 
Los estudios de tipo ultraestructural, en nuestro medio, son escasos debido a las dificultades en la manipulación y altos costos de la microscopía electrónica. Las técnicas descritas para el procesamiento de hongos para microscopía electrónica de rastreo (MER) presentan pocas modificaciones del esquema general, que incluye una fijación con aldehídos, una postfijación con $\mathrm{OsO}_{4}$, deshidratación, secado y cobertura iónica (5).

En este trabajo se empleó la fijación con vapores de acroleína y secado en alto vacío para el estudio ultraestructural de las características morfológicas de esporulación de algunos de los principales hongos potencialmente alergénicos encontrados en la atmósfera de Santafé de Bogotá, por estudios aerobiológicos previos de deposición atmosférica (1).

\section{Materiales y métodos}

Hongos: se utilizaron cepas colombianas de origen ambiental de Cladosporium sp., Aspergillus flavus, Aspergillus niger, Aspergillussp., Penicilliumsp., Epicoccum nigrum y Alternaria tenuis, conservadas liofilizadas en la micoteca del Centro de Investigaciones Microbiológicas de la Universidad de Los Andes, Santafé de Bogotá.

Cultivos: las cepas liofilizadas se reconstituyeron con $3 \mathrm{~mL}$ de caldo de Sabouraud (SAB) y se subcultivaron en agar $S A B$ a $22^{\circ} \mathrm{C}$. A partir de cada cepa se realizaron 20 microcultivos (6), en papa dextrosa agar (PDA), cinco en portaobletos para evaluar la esporulación en el microscopio de luz (ML) y 15 utilizando una placa metálica pequeña estéril, que sirve de soporte y base para procesamiento al MER.

Evaluación de la esporulación: se realizó una observación general del crecimiento de los cultivos en lámina portaobjetos en un microscopio estereoscópico cada $72 \mathrm{~h}$ después de la siembra inicial y se evaluó la esporulación en microscopio de luz por medio de montajes en azul de lactofenol (6).

Procesamiento y observación al MER: una vez se obtuvo una óptima esporulación, se desprendió la lámina metálica del microcultivo. El crecimiento obtenido sobre la cara inferior se sometió a vapores de acroleína pura por tres horas; se postfijó con cristales de $\mathrm{OsO}_{4}$ por $12 \mathrm{~h}$ y se cubrió con una ultracapa de $200 \AA$ de oro en un cobertor iónico (EIKO IB-3) por dos minutos (0,05 Torr, 10 $\mathrm{mA}, 350 \mathrm{Kv}$ ) para aumentar su reflectividad electrónica (comunicación personal de Yutaka Futaesaku, Universidad de Kitasato, Japón). La observación se realizó en un MER Hitachi S-570 a $15 \mathrm{Kv}$ y una resolución de $30 \AA$. Las fotos se tomaron a una distancia de trabajo entre 1 y 11 $\mathrm{mm}$, con inclinaciones entre 0 y $45^{\circ}$, entre 500 y 15.000 aumentos, utilizando película Kodak VP120 (Verichrome pan film) ISO 125.

\section{Resultados}

Las micrografías 1 y 2 corresponden a un Penicillium sp., monoverticilado; se observa el conidióforo, las fiálides (célula conidiógena) en forma de frasco y los conidios en sucesiones basipétalas, conidio joven naciendo en cadena en el ápice de la fiálide. Un acercamiento permite apreciar con detalle la superficie rugosa de los conidios observándose las uniones que permiten el arreglo de los conidios en sucesión basipétala (micrografía 3).

Aspergillus flavus muestra el conidióforo y la vesícula apical, ambos rugosos, y los arreglos morfológicos característicos de este hongo, observándose claramente que la zona fértil se encuentra en la mitad superior de la vesícula apical (micrografía 4) o rodeando toda la superficie (micrografía 5). Un acercamiento permite apreciar la superficie espinosa de los conidios (micrografía 6).

En las micrografías 7 y 8 se observa que la zona fértil de Aspergillus niger se encuentra localizada en toda la superficie de la vesícula apical a la vez que se observan conidióforos lisos; un acercamiento de los conidios muestra la superficie espinosa y la disposición basipétala característica se aprecia en la micrografía 9. Aspergillus sp. muestra un conidióforo de tipo rugoso, apreciándose fiálides biseriadas sobre toda la vesícula apical (micrografías 10 y 11), que probablemente permitan ubicarlo en los grupos flavus-oryzae, ochraceus o tamarii. 

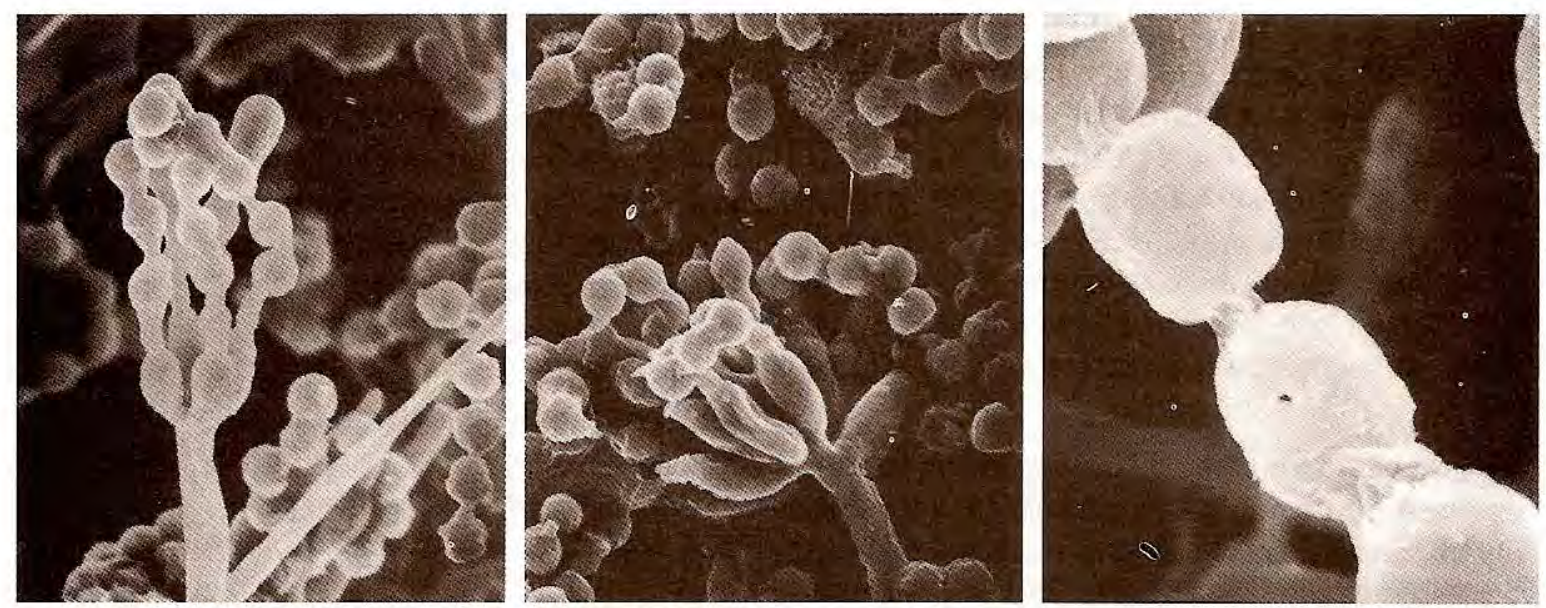

Micrografías 1-3. Penicillium sp.
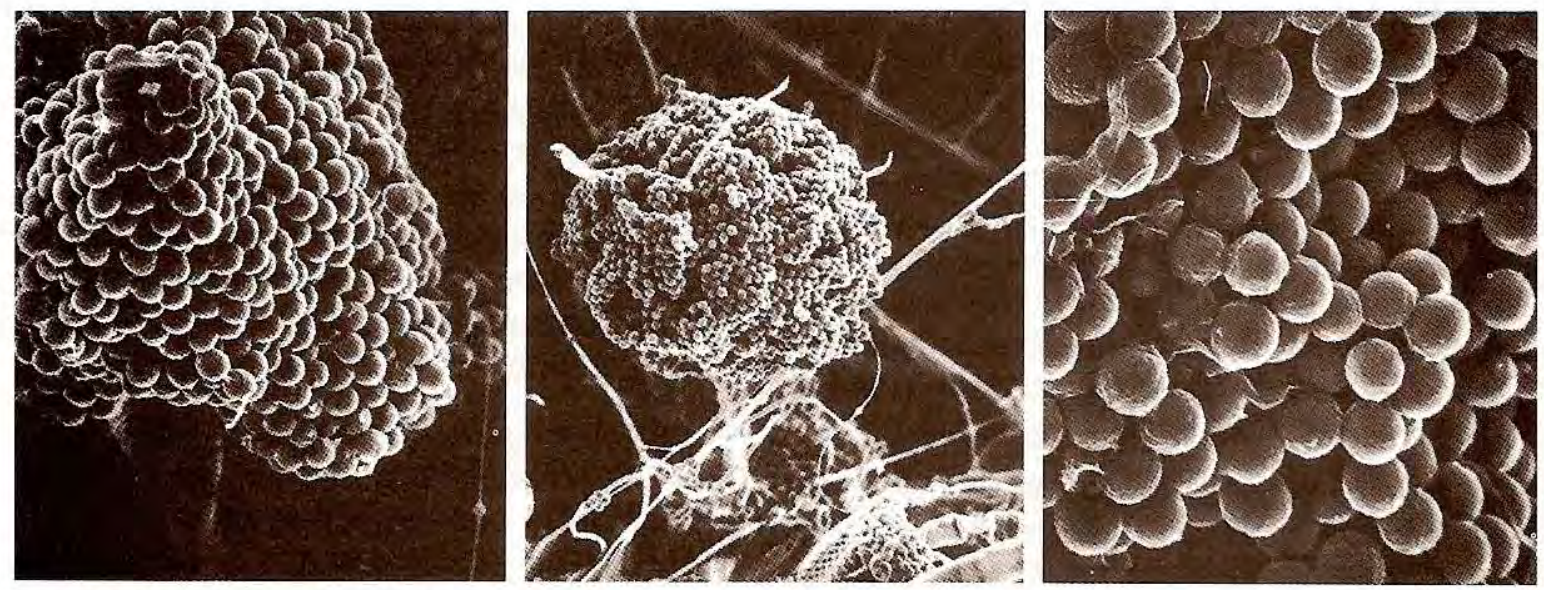

Micrografías 4-6. Aspergillus flavus.
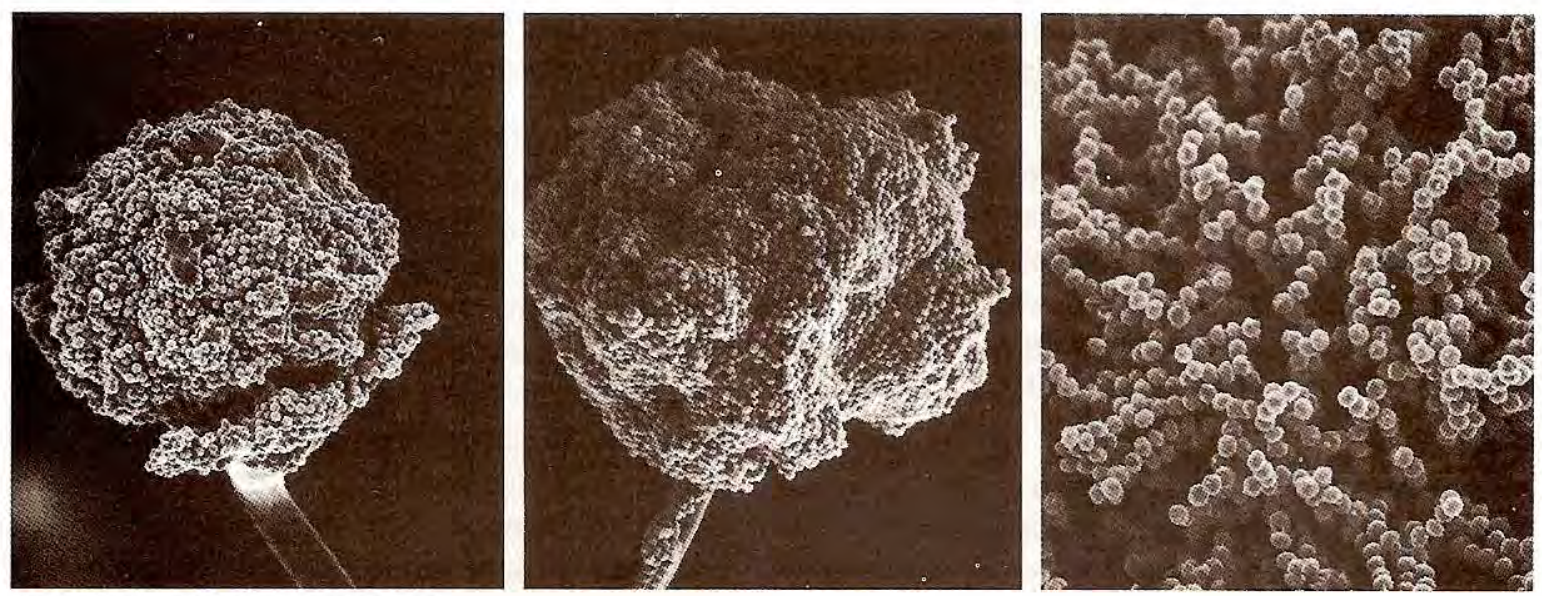

Micrografías 7-9. Aspergillus niger. 

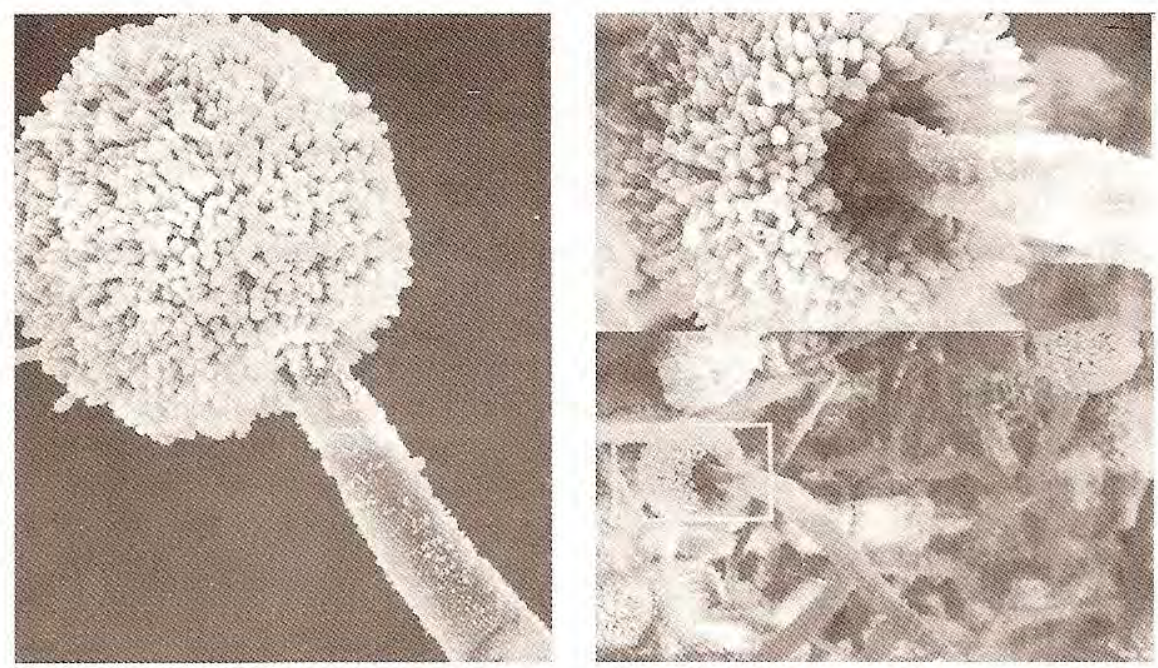

Micrografías 10-11.

Aspergillus sp.

Micrografías 12-15. Cladosporium sp.
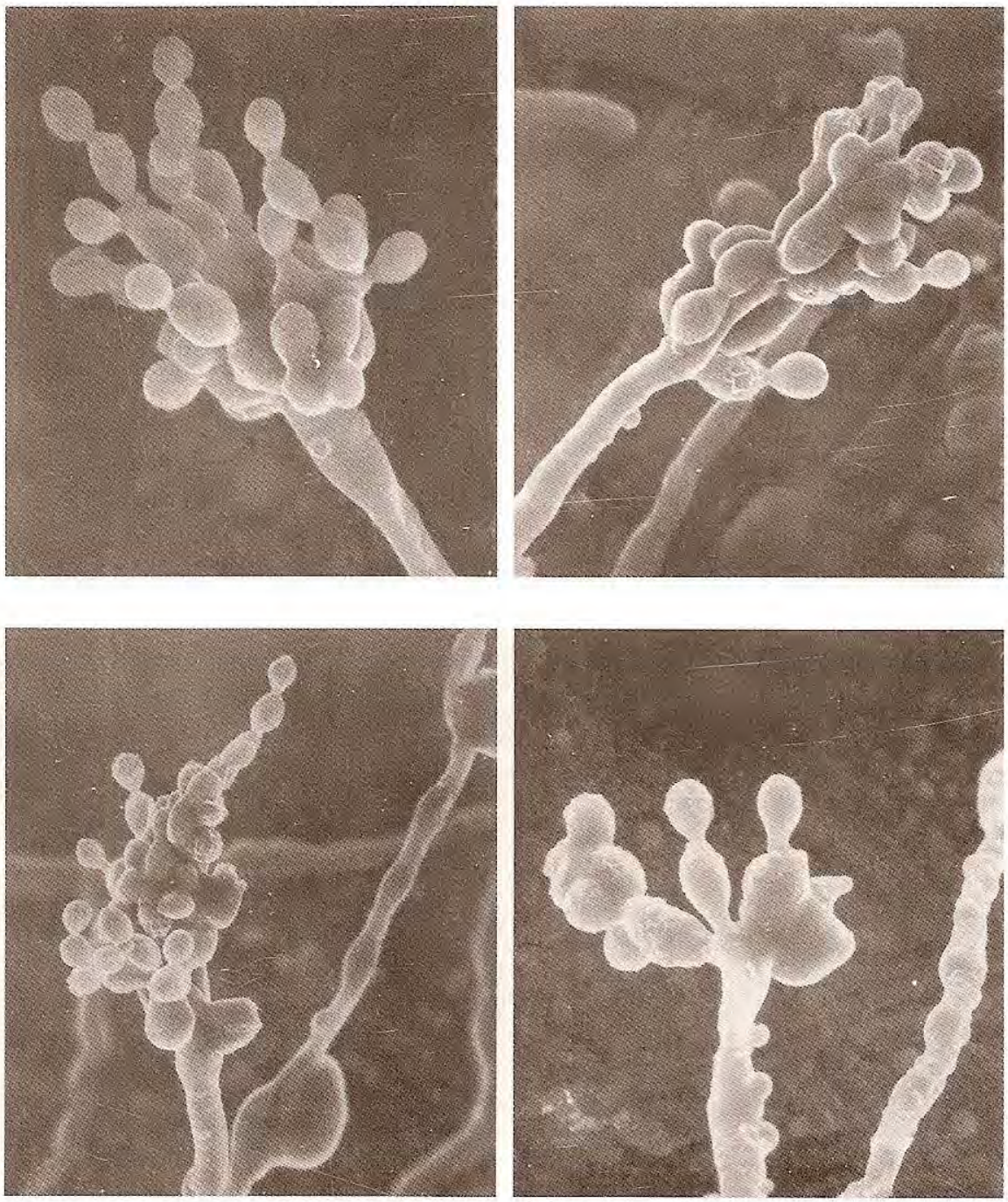
La cepa de Cladosporium sp. muestra conidiogénesis poliblástica, conidióforo diferenciado simple liso (micrografías 12-14) o con nódulos (micrografía 15); las cadenas acropétalas de conidios reticulados son producidos de manera más o menos simpodial en forma terminal o intercalar, que probablemente permitan identificarlo como Cladosporium herbarum. Nótese los dentículos o cicatrices, puntos fértiles de donde se originan otros conidios (micrografía 12).

En la cepa de Epicoccum nigrum se pueden apreciar conidios globosos de superficie verrucosa en diferentes estados de desarrollo (micrografía 16), agregados dentro de densas masas de esporodoquios en formación, característico de la conidiogénesis de esta especie. Se observan algunos conidióforos cortos con el conidio en sus primeros estados de formación (micrografía 17).

Alternaria tenuis presenta en la micrografía 18 un conidióforo recto, lateral, liso, corto, simple, diferenciado, naciendo de una hifa no diferenciada de superficie nudosa, observándose en la superficie apical del conidio la formación de uno nuevo en cada acropétala. Se observan también conidios solitarios, ovoides, piriformes de superficie rugosa, con ápice liso (micrografía 19), apreciándose el poroconidio en el extremo basal (micrografía 20).
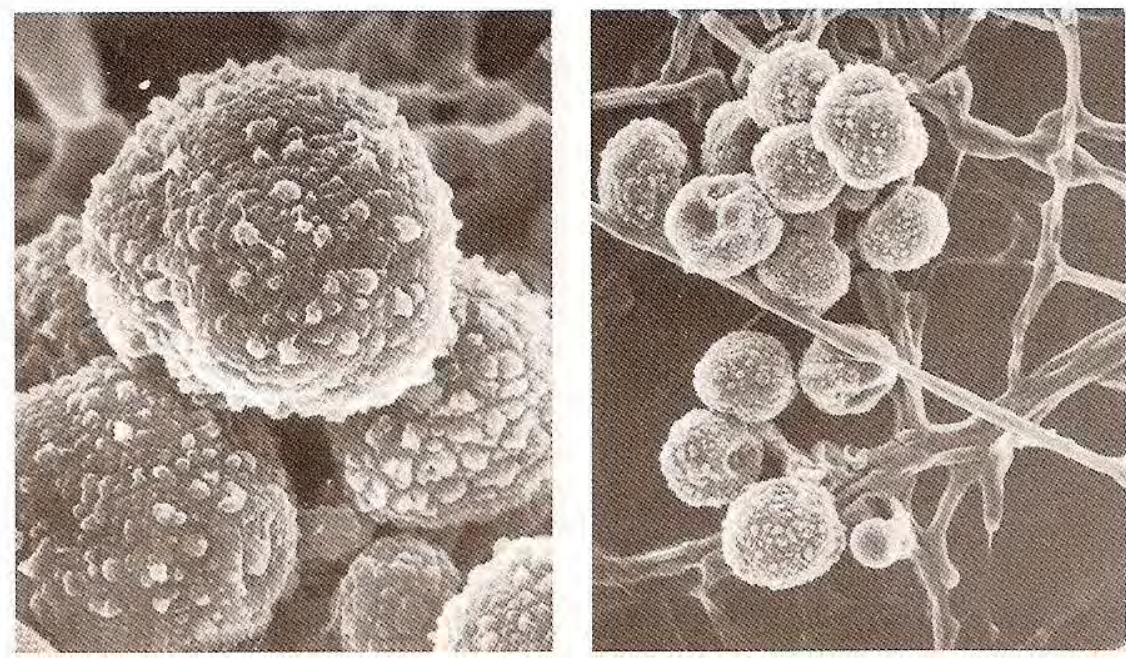

Micrografías 16-17. Epicoccum nigrum.
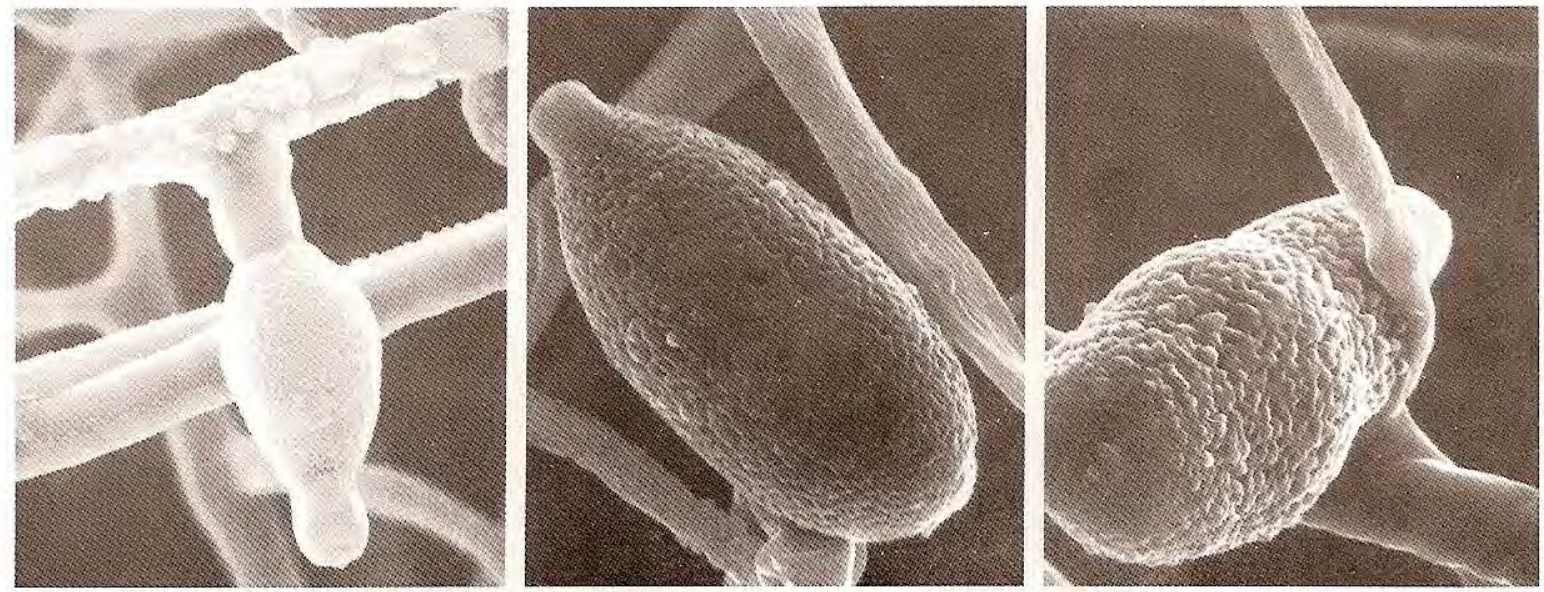

Micrografías 18-20. Alternaria tenuis. 


\section{Discusión}

El conocimiento de la ultraestructura de los hongos es paralelo al desarrollo del microscopio electrónico y de las técnicas empleadas para su procesamiento. El microscopio de luz ha contribuido en gran parte al estudio de la conidiogénesis, germinación, interacción huésped-patógeno y organización funcional y estructural (7-9).

Existen varios métodos descritos para el procesamiento de hongos al MER (8-10) los cuales son decisivos para obtener un buen análisis ultraestructural; una deficiencia en el procesamiento, repercute en la buena interpretación de la imagen. El método empleado en este trabajo ha mostrado ventajas sobre otros métodos convencionales de procesamiento (11); mantuvo intactas las estructuras aéreas y arreglos característicos de los hongos analizados, así como ya lo hemos informado en un estudio previo sobre Fonsecaea pedrosoi (2).

Las características y el poder de resolución del MER, así como el método empleado, permitieron examinar el conidióforo entero con sus células conidiógenas, conidios y estructuras adyacentes, revelando estructuras morfológicas diversas que aunque no difieren de las observaciones hechas con microscopio de luz y con microscopio de contraste de fases, sí permiten la obtención de detalles más completos de la superficie externa de los hongos estudiados y de la uniones entre las células, como los observados en la micrografía 3 , no visibles con otros tipos de microscopios.

Así mismo al microscopio de luz no se define la superficie reticulada de los conidios de Cladosporium sp. y es de difícil observación la formación de esporodoquios en E. nigrum. Estas características de interés taxonómico, sólo se evidenciaron al MER. Además, los dentículos, puntos fértiles o cicatrices de esporulación, y la superficie rugosa de los dictioconidios y nódulos en las hifas de Alternaria tenuis son también de difícil observación al microscopio de luz. En $A$. tenuis no se observó la producción simpodial de los conidios ni las cadenas características; se detallan el conidióforo simple y los conidios solos y no las cadenas características de conidios muriformes septados, probablemente, por el tiempo de incubación de la cepa estudiada.

La apreciación de estos detalles ultraestructurales al MER ayudan a un mejor entendimiento del proceso de la conidiogénesis y son útiles en la realización de estudios morfológicos, estructurales y taxonómicos.

En el estudio de la micología médica es cada día más necesario el conocimiento profundo de los hongos encontrados en el medio ambiente debido a que son potentes alergenos que causan en personas susceptibles estados severos de hipersensibilidad $(12,13)$. Actualmente, se observa un incremento de micosis sistémicas y oportunistas por algunos de estos hongos en pacientes con tratamiento citostático (14) e inmunosupresor (15), así como en pacientes con el síndrome de inmunodeficiencia adquirida (1618) y queratitis micótica (comunicación personal de María C. de García, CIMIC, Universidad de Los Andes).

La presencia de estos hongos en el medio ambiente está determinada por factores climatológicos, como estaciones y épocas de lluvias, y factores ecológicos del hábitat, los cuales presentan variaciones de un sitio a otro. Todo esto establece un ritmo en la contaminación ambiental, siendo posible establecer calendarios aerobiológicos estudiando durante un año un determinado lugar de interés para conocer sus características ecológicas específicas (1).

\section{Agradecimientos}

A la Agencia de Cooperación Internacional del Gobierno del Japón (JICA), a la Unidad de Microscopía Electrónica de la Universidad de Costa Rica y a los doctores Yutaka Futaesaku y Myrtha Arango por sus valiosas sugerencias.

\section{Referencias}

1. Hurtado I, Leal F, Rodríguez A, García E, Alson-Jaran J. A one year survey of airbone pollen and spores in the neotropical city of Bogotá (Colombia). Allergol Immunopathol 1989;17:95-104.

2. Yung J, García M, Leal F. Comparación de algunos métodos de procesamiento para Fonsecaea pedrosoi al microscopio electrónico de rastreo. Biomédica 1995;1:21-8. 
3. Burge HA, Fungus allergens. Clin RevAllergy 1985;3:319.

4. Greenberger PA, Paterson R. Allergic bronchopulmonary aspergillosis and the evaluation of the patient with asthma. J Allergy Clin Immunol 1988;81:646.

5. Arroyo 0. Conceptos básicos sobre el procesamiento de tejidos para microscopía electrónica. San José: Editorial de la Universidad de Costa Rica, 1991.

6. Konemam EW, Robert G. Micología práctica de laboratorio. Tercera edición. BuenosAires: Editorial Médica Panamericana, 1987:92-93.

7. Shapiro J. Organization of developing Escherichia coli colonies viewed by scanning electron microscopy. $\mathrm{J}$ Bacteriol 1987;169:142-156.

8. Mares D. Electron microscopy of Microsporum cokkei after in vitro treatment with protoanemonium: a combined SEM and TEM study. Mycopathologia 1989;108:37-46.

9. Klomparens K. The development and aplication of structural research in mycology. Mycopathologia 1990;109:139148.

10. Cole GT. Conidiogenesis and coniomatal ontogeny. In: Cole GT, Kendrick B, editors. Biology of conidial fungi. Vol. 2. New York: Academic Press, 1980:261-7.

11. Agudelo CM, Chica J. Procesamiento de hongos para microscopía electrónica de rastreo a partir de micro- cultivos. Evaluación de los métodos descritos (Tesis). Santafé de Bogotá, D.C.: Universidad de LosAndes, 1992.

12. Rippon LW. The pathogenic fungi and the pathogenic actinomycetes. Second edition. Philadelphia: WB Saunders, 1982:714-8.

13. Gumowsky D, Latgé JP, Paris S. Fungal allergy. In:Arora DK, Ajello L, Mukerji JG, editors. Handbook of applied mycology: humans, animals, and insects. Vol. 2. New York: Marcel Dekker Inc., 1991:163-204.

14. Anaissie E. New spectrum of fungal infection in patients with cancer. Rev Infect Dis 1989;11:369-78.

15. Body BA. Alternaria species infection in a patient with acute lymphocytic leukemia. Pediatr Infect Dis J 1987;6:418-9.

16. Wiest PM. Alternaria infection in a patient with acquired immunodeficiency syndrome: case report and review of invasive Alternaria infections. Rev Infect Dis 1987;9:799803.

17. Alvarez S. Systemic infection caused by Penicillium decumbens in a patient with acquired immunodeficiency syndrome. J Infect Dis 1990;162:283.

18. Lortholary O, Meyohas MC, Dupont B, et al. Invasive aspergillosis in patients with acquired immunodefiency syndrome: report of 33 cases. Am J Med 1993;95:177-87. 